\title{
Article
}

http://dx.doi.org/10.11646/phytotaxa.172.3.1

\section{Elucidating the controversial Drosera montana complex (Droseraceae): a taxonomic revision}

\author{
FERNANDO RIVADAVIA ${ }^{1}$, PAULO MINATEL GONELLA ${ }^{2 *}$, PAULO TAKEO SANO ${ }^{3} \&$ ANDREAS FLEISCHMANN $^{4}$ \\ ${ }^{1}$ Daniel Burnham Ct., San Francisco, 94109,_USA; e-mail:fe_riva@uol.com.br \\ ${ }^{2}$ Programa de Pós-Graduação em Botânica, Laboratório de Sistemática Vegetal, Departamento de Botânica, Instituto de Biociências, \\ Universidade de São Paulo, CEP 05508-900,São Paulo,Brasil; e-mail: paulogonella@uol.com.br \\ ${ }^{3}$ Laboratório de Sistemática Vegetal, Departamento de Botânica, Instituto de Biociências, Universidade de São Paulo, CEP 05508-900, \\ São Paulo,Brasil; e-mail: ptsano@usp.br \\ ${ }^{4}$ Botanische Staatssammlung München, Menzinger Strasse 67, D-80638 Munich, Germany; e-mail: fleischmann@lrz.uni-muenchen.de \\ * author for correspondence
}

\begin{abstract}
The species of the affinity of Drosera montana (Droseraceae) are reviewed taxonomically and the complex is redefined to include only D. montana, D. tentaculata, D. tomentosa var. tomentosa, D. tomentosa var. glabrata, and D. spirocalyx. The latter is a newly described narrow endemic species from the Serra do Cipó in central Minas Gerais state, Brazil. The morphological characters distinguishing these five taxa from each other and from other similar species are discussed together with habitat and ecological information. Detailed illustrations, photographs, distribution maps and an identification key are provided. A lectotype for $D$. tomentosa is here designated.
\end{abstract}

Keywords: Brazil, carnivorous plants, Drosera tentaculata, Drosera tomentosa, Drosera tomentosa var. glabrata, Drosera spirocalyx, ecology, morphology, new species, lectotypification, taxonomy

\section{Resumo}

As espécies do complexo Drosera montana (Droseraceae) são revistas taxonomicamente e o complexo é redefinido, passando a incluir apenas $D$. montana, D. tentaculata, D. tomentosa var. tomentosa, D. tomentosa var. glabrata e D. spirocalyx. A última é uma espécie aqui descrita pela primeira vez, sendo endêmica da Serra do Cipó, região central de Minas Gerais, Brasil. Os caracteres morfológicos distinguindo estes cinco táxons entre si e de outras espécies similares são discutidos junto a informações sobre habitat e ecologia. Ilustrações detalhadas, fotografias, mapas de distribuição e uma chave são apresentados. Um lectótipo de D. tomentosa é aqui designado.

Palavras-chave: Brasil, Drosera tentaculata, Drosera tomentosa, Drosera tomentosa var. glabrata, Drosera spirocalyx, ecologia, morfologia, novas especies, lectotipificação, plantas carnívoras, taxonomia

\section{Introduction}

The species complex of the Drosera montana Saint-Hilaire (1826: 260) affinity currently comprises a confusing list of 16 names proposed over nearly 200 years of taxonomic research on the genus Drosera in Brazil. This was initiated by the French naturalist Auguste de Saint-Hilaire $(1824,1826)$ who described the first Brazilian Drosera taxa. Since then, most botanists have applied a rather expansive taxonomic circumscription of $D$. montana, resulting in a classification that synonymized under this taxon several previously segregated species (e.g. Diels 1906, Correa \& Silva 2005). The history of taxa associated with the D. montana complex is summarized below:

- $\quad$ Saint-Hilaire (1826) described D. montana, D. parvifolia Saint-Hilaire (1826: 263), D. tomentosa Saint-Hilaire (1826: 261) var. tomentosa, D. tomentosa var. glabrata Saint-Hilaire (1826: 262), D. hirtella Saint-Hilaire (1826: 262) var. hirtella, and D. hirtella var. lutescens Saint-Hilaire (1826: 263), together with six other Drosera taxa of different affinities (see Gonella et al. 2012, 2014). 


\section{References}

Brako, L. \& Zaruchi, J.L. (1993) Catalogue of the flowering plants and gymnosperms of Peru. Monographs in Systematic Botany from theMissouri Botanical Garden 45: 1-1286.

Brummer-Dinger, C.H. (1955) Notes on Guiana Droseraceae. Acta Botanica Neerlandica 4(I): 136-138. http://dx.doi.org/10.1111/j.1438-8677.1955.tb00321.x

Chodat, R. (1903) Droseraceae In: Chodat, R. \& Hassler, E. (eds.) Plantae Hasslerianae soit énumération des plantes récoltées au Paraguay. Bulletin de l'Herbier Boisier II(3): 538-552. http://dx.doi.org/10.5962/bhl.title.45112

Correa A., M.D. \& Silva, T.R.S. (2005) Drosera (Droseraceae). Flora Neotropica Monograph 96. The New York Botanical Garden Press, Bronx, NY, 65 pp.

Correa A., M.D. \& Taylor, B. (1977) Flora of Panama: V. Family 76A. Droseraceae. Annals of the Missouri Botanical Garden 63: 389-392. http://dx.doi.org/10.2307/2395279

Diels, L. (1906) Droseraceae. In: A. Engler. (ed.) Das Pflanzenreich IV.112 (Heft 26). W. Engelmann, Leipzig, pp 1-136.

Diels, L. (1914) Droseraceae. In: Pilger, R (ed.), Plantae Uleanae novae vel minus cognitae 1: 136. Botanischer Garten und Botanisches Museum, Berlin-Dahlem.

Duno de Stefano, R. \& Silva, T.R.S. (2001) Conservation status of the carnivorous plant genus Drosera in South America and the Caribbean. Harvard Papers in Botany 6: 253-260.

Eichler, A.G. (1872) Droseraceae. In: Martius, C.F.P. von \& Eichler, A.G. (eds.) Flora Brasiliensis 14(2). Typographia Regia, München, pp. 385-398.

ESRI (2011) ArcGIS Desktop: Release 10. Redlands, CA: Environmental Systems Research Institute.

Fernández-Pérez, A. (1965) Plantas Insectívoras, II Droseraceae de Colombia. Caldasia 11: 219-232.

Ferrero, R. \& Mello-Silva, R. (2011) Droseraceae do Parque Estadual do Ibitipoca, Minas Gerais, Brasil. Boletim de Botânica da Universidade de São Paulo 29(1): 13-18.

http://dx.doi.org/10.11606/issn.2316-9052.v29i1p13-18

Fleischmann, A., Wistuba, A., McPherson, S. (2007) Drosera solaris (Droseraceae), a new sundew from the Guayana Highlands. Willdenowia 7: 551-555. http://dx.doi.org/10.3372/wi.37.37214

Gonella, P.M., Rivadavia, F. \& Sano, P.T. (2012) Re-establishment of Drosera spiralis (Droseraceae), and a new circumscription of $D$. graminifolia. Phytotaxa 75: 43-57. http://dx.doi.org/10.11646/phytotaxa.75.1.4

Gonella, P.M., Rivadavia, F., Sano, P.T. \& Fleischmann, A. (2014) Exhuming Saint-Hilaire: revision of the Drosera villosa complex (Droseraceae) supports 200 year-old neglected species concepts. Phytotaxa 156(1): 1-40. http://dx.doi.org/10.11646/phytotaxa.156.1.1

Hartmeyer, I. \& Hartmeyer S. (2006) Clandestine diversity: snap-tentacles of the genus Drosera. Carniflora Australis 7: 4-18.

Hooker, J.D. (1852) Nat. Ord. V. Droseraceae, DC. In: Hooker, J.D. (ed.) Botany of the Antarctic Voyage 2: Flora novae-zelandiae, Part 1. Reeve Brothers, London, pp. 19-21.

IUCN (2001) IUCN red list categories and criteria: Version 3.1. IUCN Species Survival Commission. IUCN, Gland, Switzerland and Cambridge, UK. Ii +30 pp.

Linnaeus, C. (1753) Species Plantarum 1. Impensis Laurentii Salvii, Holmiae, 572 pp.

Maguire, B. \& Laundon, J.R. (1957) Drosera roraimae. In: Maguire, B. \& Wurdack, J.J. (eds.) The Botany of the Guayana Highlands - Part II. Memoirs of the New York Botanical Garden 9(3): 33-34.

Maguire, B. \& Wurdack, J.J. (1957) The botany of the Guayana Highland, Part II. Memoirs of the New York Botanical Garden 9: 331336.

Pignal, M., Romaniuc-Neto, S., De Souza, S., Chagnoux, S. \& Lange Canhos, D.A. (2013) Saint-Hilaire virtual herbarium, a new upgradeable tool to study Brazilian botany. Adansonia, séries 3, 35 (1): 7-18.

http://dx.doi.org/10.5252/a2013n1a1

Poiret, J.L.M. de (1804) Rossolis. Drosera. In: Poiret, J.L.M. de (ed.) Encyclopedie Methodique. Botanique 6(1). Chez Panckoucke, Paris, pp. 298-302.

Pursh, F.T. (1813) Drosera. In: Pursh, F.T. (ed.) Flora Americae Septentrionalis, or a systematic arrangement and description of the plants of North America 1. White, Cochrane and Co., London, pp. 210-211.

http://dx.doi.org/10.5962/bhl.title.6122

Rivadavia, F. (1999) Neblina expedition part 5. Listserve Archives of the Carnivorous Plant Discussion Group Mailing List. Available 
from: http://www.omnisterra.com/botany/cp/list/cp99all.d/0609.htm (accessed 22 October 2013).

Rivadavia, F. (2003) Four new species of sundews, Drosera (Droseraceae), from Brazil. Carnivorous Plant Newsletter 32: 79-92.

Rivadavia, F. (2005) New chromosome numbers for Drosera L. (Droseraceae). Carnivorous Plant Newsletter 34: 85-91.

Rivadavia, F. (2008) The Drosera montana A.St.-Hil. (Droseraceae) complex: a new combination, Drosera schwackei (Diels) Rivadavia, is proposed. Carnivorous Plant Newsletter 37: 36-43.

Rivadavia, F. (2009) Drosera x fontinalis (Droseraceae), the first natural sundew hybrid from South America. Carnivorous Plant Newsletter 38: 121-125.

Rivadavia, F. \& Gonella, P.M. (2011) Drosera quartzicola (Droseraceae), a new and threatened species from the Serra do Cipó, Brazil. Phytotaxa 29: 33-40.

Rivadavia, F., Kondo, K., Kato, M. \& Hasebe, M. (2003) Phylogeny of the sundews, Drosera (Droseraceae), based on chloroplast rbcL and nuclear 18S ribosomal DNA sequences. American Journal of Botany 90: 123-130.

http://dx.doi.org/10.3732/ajb.90.1.123

Rivadavia, F., Miranda, V.F.O., Hoogenstrijd, G., Pinheiro, F., Heubl, G. \& Fleischmann, A. (2012) Is Drosera meristocaulis a pygmy sundew? Evidence of a long-distance dispersal between Western Australia and northern South America. Annals of Botany 110: 11-21. http://dx.doi.org/10.1093/aob/mcs096

Saint-Hilaire, A.F.C.P. de (1824) Plantes usuelles des brasiliens 3. Grimbert, Paris, 300 pp.

Saint-Hilaire, A.F.C.P. de (1826) Histoire des plantes les plus remarquables du Brésil et du Paraguay 1(6). A. Belin, Paris [exact content of part 6 unknown].

Santos, E. (1986) O gênero Drosera L. no Brasil I - uma nova espécie. Bradea 4(38): 305-308.

Santos, E. (1989) O gênero Drosera L. no Brasil II - sobre D. montana St.-Hil. Bradea 5(21): 249-256.

Schulenberg, T. S. \& K. Awbrey. (1997) A rapid assessment of the humid forests of South Central Chuquisaca, Bolivia. RAP Working Papers 8: 1-84.

Seine, R. \& Barthlott, W. (1993) On the morphology of trichomes and tentacles of Droseraceae Salisb. Beitrage zur Biologie der Pflanzen 67: 345-366.

Silva, T.R.S. (1994) Estudos Taxonômicos de Drosera (Droseraceae) do Brasil. Master thesis. Universidade de São Paulo, São Paulo, Brazil, 127 pp.

Silva, T.R.S. (1997) Drosera graomogolensis (Droseraceae), a new species from the campos rupestres of Minas Gerais, Brazil. Novon 7: 85-87. http://dx.doi.org/10.2307/3392080

Silva, T.R.S. (2002) Droseraceae. In: Wanderley, M.G.L., Sheperd, G.J., Giulietti, A.M., Melhem, T.S., Volker, B. \& Kameyama, C. (eds.) Flora Fanerogâmica do Estado de São Paulo 2 FAPESP/HUCITEC, São Paulo, pp.101-102.

Silva, T.R.S. (2007) Flora da Bahia - Droseraceae Salisb. Sitientibus Série Ciências Biológicas 7(4): 393-397.

Silva, T.R.S. (2013) Droseraceae In Lista de Espécies da Flora do Brasil. Jardim Botânico do Rio de Janeiro. Available from: http:// floradobrasil.jbrj.gov.br/jabot/floradobrasil/FB105 (accessed 03 June 2013).

Silva, T.R.S. \& Giulietti, A.M. (1997) Levantamento das Droseraceae do Brasil. Boletim de Botânica da Universidade de São Paulo 16: 75-105.

http://dx.doi.org/10.11606/issn.2316-9052.v16i0p75-105

Steyermark, J.A. \& Smith, L.B. (1974) A new Drosera from Venezuela. Rhodora 76: 491-493.

Taubert, P. (1893) Plantae Glaziovianae novae vel minus cognitae. Botanische Jahrbücher für Systematik, Pflanzengeschichte und Pflanzengeographie 17: 502-506.

Thiers, B. (2011) Index herbariorum, a global directory of public herbaria and associated staff. New York Botanical Garden's Virtual Herbarium. Available from: http://sweetgum.nybg.org/ih/ (accessed: 09 October 2013). 